\title{
Software applications complexity evaluation in a bank environment
}

\author{
D. Cellino \\ Banksiel s.p.a. \\ c.so Galileo Ferraris 86 - Torino - Italy \\ tel. +39-11-505921 fax : +39-11-505164
}

\begin{abstract}
In an effort to establish a software measurement activity in a bank environment, we started considering Halstead's Software Science. We soon recognised we needed 'application metrics' more than 'program metrics' and 'user metrics' more than 'classic metrics'. This paper describes our results in validating and tailoring Halstead's Software Science in our environment and our approach in defining application and user metrics.
\end{abstract}

\section{Keywords}

Complexity measures, software applications metrics, metrics validation, bank software, McCabe metrics, Software Science.

\section{INTRODUCTION}

In 1994 an important Italian bank began to set up a measurement program to evaluate the quality of its software, with the objective to :

- keep track of time evolution of different metrics during the software life cycle;

- find metrics to measure applications and not only programs;

- find a way to measure not only the 'intrinsic' complexity of a software module but as part of an application;

- identify particularly complex software modules within a specific application.

My team and I began gathering data on the source programs using the McCabe's cyclomatic number and Halstead's Software Science metrics which are widely accepted and easy to evaluate. But, as the data were collected, it became quite clear that they did not portray 
accurately bank software quality and that, to characterise it more precisely, it should have been necessary to identify metrics more tailored on our specific environment.

This was not all that surprising since both McCabe and Halstead analyses were developed in the seventies in a completely different technical setting. After discovering, in the hard way, the drawbacks of classic metrics, we tried to find our own answers to our own software measurement problems.

We chose to focus on size and complexity of the programs and on the type of 'call statements' used", regarding these characteristics as indicators of maintainability and readability. Since the bank was only concerned about the software developed and maintained by its own personnel, software purchased under a license agreement has not been considered in our work. The study considered a set of $617 \mathrm{COBOL}$ and $64 \mathrm{PL} / \mathrm{I}$ programs belonging both to 'new' and 'old' applications, written with different techniques and affected by different amount of maintenance activity. Both 'on line' and 'batch'" programs were considered and their behaviours were investigated separately during the study.

'Copy' and 'include' statements were expanded before analysing the software to allow a complete evaluation of the programs and of the effort required to fully understand them.

The software analyser was configured to equally rate constructs which are equivalent in the two languages. For instance, the statements

- $\mathrm{A}=\mathrm{B}$

- move $\mathrm{A}$ to $\mathrm{B}$ (COBOL style assignment)

are both counted as made up of one operator and two operands.

The final result of this work has been a software application that analyses sources, evaluates several new 'customised' metrics, stores metrics history for each module and application and performs some statistical analysis to find out the acceptability ranges for modules metrics within a specific application.

\section{USING CLASSICAL METRICS}

The main drawback of McCabe's cyclomatic number is its lack of resolution : too many (and too different) programs have the same cyclomatic number. This occurs because this index counts the number of nodes in the graph regardless of their relative disposition, which is obviously where program complexity actually lies.

Other complexity metrics (for instance, Belady's bandwidth metric, Chen's entropy or the chunk-oriented measures) pay attention to the nesting levels and differentiate between nested decisional statements and sequential ones, allowing finer classifications; of course these metrics

\footnotetext{
"explicitly referring to the called module or getting its name from a program variable, thus making the program flow less obvious to understand.

"A program is 'on line' if it processes a single data item in real time (typically interfacing a video unit or a printer); it is 'batch' if it processes 'as soon as possible' (i.e. not in real time) large amounts of data typically producing a file or a printed report.
} 
are much more difficult to evaluate.

As far as Halstead's metrics (Halstead, 1977) are concerned, some of them show a curious property which questions their usefulness (Weyuker, 1988).

For instance, one would expect the effort and time required to develop a program to be greater than those needed to develop its initial portion : the opposite would be very hard to justify, but under certain conditions Halstead's effort shows this peculiar behaviour.

This occurs because the effort is defined by Halstead as a monotonically increasing function of all the basic counters (total and distinct operators $N_{1}$ and $n_{1}$ and total and distinct operands $\mathrm{N}_{2}$ and $\mathrm{n}_{2}$ ) except the number of distinct operands $\mathrm{n}_{2}$. Since the whole program will probably contain more distinct operands than its initial portion, it is not difficult to find pieces of code for which the effort decrease as the program grows.

And since Halstead's programming time is given by the Effort/Stroud number (the number of elementary discrimination per second), for such programs the time needed for developing the initial part would be greater than that needed for finishing it, which is clearly paradoxical.

We decided, before going on with a large scale measurement activity, to better investigate also some less obvious metrics proposed by Halstead.

We began working on such indicators as language level, program level, bugs number and intelligence content.

We started from the language relationship :

$$
\lambda=\mathrm{L}^{2} \mathrm{~V}
$$

which is supposed to hold between the language level $\lambda$ and the program level $\mathrm{L}$.

To verify this relationship, we estimated the best-fit value of exponent $b$ in the relationship :

$$
\lambda=\mathrm{L} b * \mathrm{~V}
$$

In 9 different sets of Cobol and PL/1 programs, we found for the exponent $b$ a set of values whose average weighed on the number of modules is $1.99 \pm 0.14$, in excellent agreement with Halstead's result.

On the other side, our study does not provide any indication that the product in (2.1) remains constant for a given language as suggested by Halstead : on the contrary, $\lambda$ shows a great variability.

It has to be noticed that our sets contain programs using CICS, DB2 or DL/1 commands, and hence it would be more appropriate to divide them in subsets according to the kind of data communication and database they use.

For instance, considering separately time-processing (CICS) programs and batch programs, the overall agreement improves, but the standard deviation remains high.

To sum up, while it is possible to say that there is a linear correlation between the logarithms of volume and program level, it seems more difficult to sustain that $\mathrm{L}^{2} \mathrm{~V}$ tends to some constant, 'fixed' for a given language.

Other Software Science indicators that we tried to validate in our environment were the potential volume of an algorithm and its 'intelligence content', which were supposed to be independent from the language in which the algorithm is expressed.

Combining the two different equations given by Halstead (Halstead, 1977) for the total 
number B of delivered bugs ${ }^{*}$, one gets :

$\mathrm{V}^{*}=\mathrm{E}^{2 / 3}$

which would give a simple way to obtain the potential volume $V^{*}$ from the effort $E$.

Unfortunately, in our study we could not find any convincing correlation between $\mathrm{V}^{*}$ and $\mathrm{E}$.

On the other side, we noticed that the intelligence content, whose complete expression is rather complicated", is very well approximated by the program vocabulary (number of distinct words used) : the correlation turned out to be 0.944 on the set of Cobol programs and 0.959 on the set of PL/1 program. This means that the intelligence content (and hence the potential volume $\mathrm{V}^{*}$ ) can be estimated by the program vocabulary.

Following the same approach, we tried to approximate the program level, the ratio between potential and real volume of the algorithm, whose value would allow to verify if the implementation of the algorithm is affected by some unnecessary complexity.

Not surprisingly the relationship between the program level and the ratio vocabulary/volume was not so good.

The correlation was still very high for Cobol (0.953), but rather low for PL/1 (0.73).

\section{CUSTOMISED PROGRAM METRICS}

Since our code analyser could not detect nested loops and structures, we were forced to set them aside (at least for the moment) as well as data flow complexity, live with McCabe and Halstead's counters and use them to define our program metrics.

We wanted also to obtain global indicators for application, a subject on which we were not able to find any help in the technical literature, and to set reasonable thresholds for module acceptability on the various metrics.

We learned from literature (McCabe, 1976) that 10 seemed 'a reasonable, but not magical, upper limit' for cyclomatic complexity and that exceptions should be made only for very wide case constructs (DeMarco, 1982). Unfortunately, this number is not reasonable at all in most of bank application software : just think of the programs which validate the customer data for the opening of an account or the mortgage payment!

A recent work by 'Corpo Tecnico dell'Esercito Italiano' (Mondino, 1994), which sets the upper limit for cyclomatic number to 15 , sets also the upper limit for the statements number to 50 which is ridiculously low for typical bank software modules.

It seems also questionable to compare 'absolute' values of cyclomatic complexity between two programs regardless of their size. If a program has 50 statements, 15 control nodes can really be too much : but what about a program with 15 control nodes and 1500 (or even 3000) statements? We did not think they could be considered 'equally complex'.

We chose Halstead's program length (the number of program words, excluding declaratives) and Halstead's program vocabulary as measures of the code size. Then, we started working not directly on counters but on their normalised form : cyclomatic numbers and total operands and operators were normalised using Halstead's program length, while distinct operands were normalised using Halstead's vocabulary. Distinct operators do not need any further

\footnotetext{
${ }^{*} \mathrm{~B}=\mathrm{E}^{2 / 3} / 3000$ and $\mathrm{B}=\mathrm{V}^{*} / 3000$

* Intelligence content $=\left(2 n_{2}\left(N_{1}+N_{2}\right) \log _{2}\left(n_{1}+n_{2}\right)\right) /\left(n_{1} N_{2}\right)$
} 
normalisation, since they are intrinsically limited by the total number of keywords of the language used to write the code.

This normalisation allowed us to compare the different values of metrics between different releases of the same program, and to understand if a particular intervention had increased or decreased its complexity, size, and data.

Some of the metrics are not related to quality aspects, but simply reflect some aspects of 'size' : for instance, the ratio 'total operands/length' can be looked at as a 'data size', which it is not per-se related to quality even if an anomalously high or low ratio could trigger further analysis.

We did not set any threshold value for 'size' metrics acceptability, while for each 'quality metric' we set two different threshold values : a 'yellow alert' and a 'red alert' threshold which partitioned the programs in three sets :

- Under the 'yellow alert' threshold :

All right (60\%)

- Above the 'yellow alert' but under the 'red alert' threshold :

- Above the 'red alert' threshold :

Could be better $(90 \%)$

Out of limits

We set these thresholds by a statistical analysis of a subset of the bank software; for some indicators, we set different thresholds for 'on line' and 'batch' programs.

'All right' does not mean that the program has really a reasonable complexity and size : it means that its metric values are under a threshold which is satisfied by the $60 \%$ of the subset used for test. The same happens to the $10 \%$ programs 'out of limits' : they are above a threshold satisfied by the $90 \%$ of the tested subset.

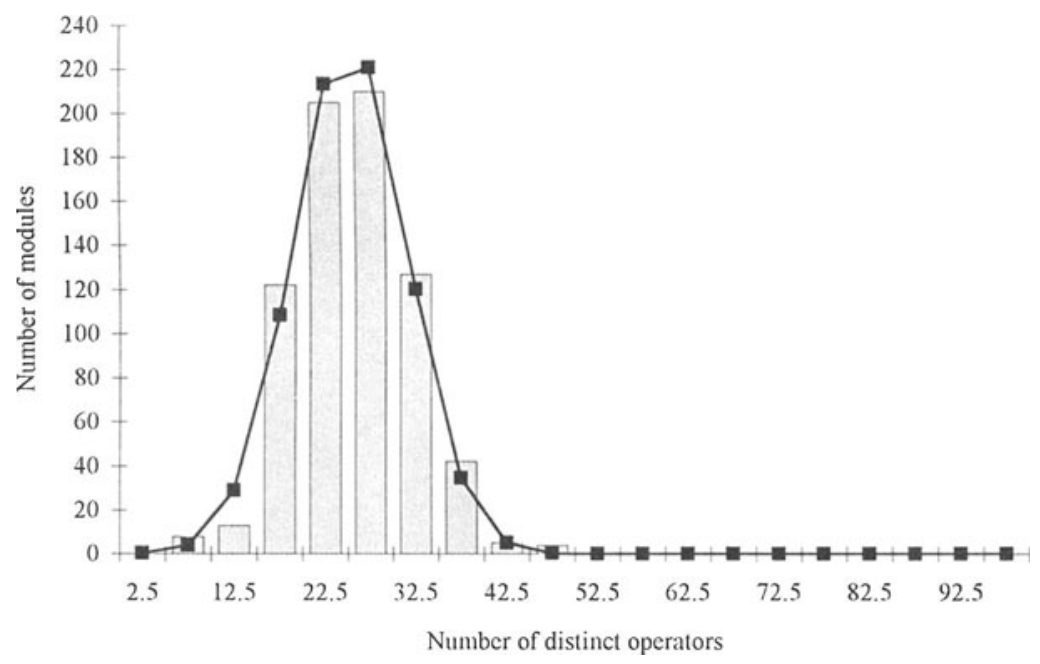

Figure 1 Frequency distribution of distinct operators (line). The bars represent the normal distribution. 
It is clear that this way of setting thresholds aims at 'relative quality'; but, on the other side, we were looking for a way to focus our maintenance activity and however it would be useless to set an upper limit of 50 statements in an environment where a program with 3000 words is 'not so big'.

A few more words about the number of distinct operators $n_{1}$. On our programs sample, we never found values higher than 50 . Moreover, we saw that the frequency distribution is close to normal (see Figure 1).

The value of $n_{1}$ lay in the range [20,30[ for 382 programs, and in the range [15,35[ for 616 programs. Only 65 programs (less than $10 \%$ ) were characterised by a $n_{1}$ greater than 35 .

Such programs could show some peculiar complexity (which required some special features of the language) or maybe simply display an unusual mastering of the language. But in any case they will be less obvious to understand for most programmers.

\section{APPLICATIONS METRICS AND EVALUATIONS OF PROGRAMS VERSUS APPLICATIONS}

Our basic assumption is that the 'computational complexity' of a program depends on the total number of operators used $\mathrm{N}_{1}$ and on the total number of 'decisional operators' TD (including logical operators like $\mathrm{OR}, \mathrm{AND}, \mathrm{NOT})$ : the higher the ratio ' $\mathrm{TD} / \mathrm{N}_{1}$ ', the more complex the program.

Examining a number of different software applications, we found out that there exists a relationship between $N_{1}$ and TD; more precisely (see Figures 2 and 3), the relationship is linear, and the linear term varies, in our study, between 0.09 and 0.2 .

The average correlation is 0.94 , which is rather good. Results are shown in Table 1 .

Table 2 makes clear that parabolic regression does not lead to better results : the square terms coefficients are negligible, and the linear terms vary in the same range found for them in linear regression. Anyway, one could choose to try parabolic regression every time linear regression proves unsatisfactory.

The results are better for $\mathrm{COBOL}$ programs than for $\mathrm{PL} / 1$ programs. We have to remark, however, the smaller dimension of the PL/1 set (only 64 programs) and the lower size of its programs.

Applications whose programs show a higher density of decisional operators will be represented by regression lines with greater linear term; thus, this parameter of the linear correlation can be chosen as a measure of application complexity.

By evaluating this factor for a greater set of applications, we are now going to establish threshold for its values, in order to rate applications as 'simple', 'normal' or 'complex'.

We have called the correlation line of an application its 'computational complexity model', which is described with three values : the linear term, the coefficient of correlation and the standard deviation of the regression.

The model is considered reliable if the coefficient of correlation is higher than $0.85{ }^{*}$

\footnotetext{
" After analysing 14342 batch programs and 9882 on-line programs, belonging to 190 different applications, we found that $96 \%$ of the on-line applications and $84 \%$ of the batch applications shows a correlation degree greater than 0.90 .
} 
Table 1 Linear regression of TD against $\mathrm{N}_{1}$

Regression line

Appl. Lang. N. pgm. Const. term Linearterm Std. err. Corr. degr.

\begin{tabular}{llrrrrr}
\hline C1 & COB & 44 & $-2,02$ & 0,151 & 5,78 & 0,93 \\
C2 & PL/1 & 36 & $-6,4$ & 0,170 & 14,4 & 0,97 \\
CTOT & Mix & 80 & $-5,27$ & 0,168 & 10,75 & 0,97 \\
CM & Mix & 4 & 32,09 & 0,149 & 42 & 0,87 \\
R1 & COB & 10 & $-16,56$ & 0,160 & 26,55 & 0,97 \\
R2 & COB & 4 & $-0,3$ & 0,137 & 1,83 & 0,99 \\
R3 & COB & 8 & $-8,69$ & 0,131 & 3,1 & 0,99 \\
R4 & COB & 5 & $-9,98$ & 0,126 & 9,76 & 0,99 \\
R5 & COB & 14 & $-10,42$ & 0,151 & 6,14 & 0,99 \\
R6 & COB & 10 & 21,57 & 0,094 & 22,36 & 0,92 \\
RB & COB & 36 & $-10,74$ & 0,151 & 16,24 & 0,98 \\
RC & COB & 15 & 10,32 & 0,108 & 21,85 & 0,95 \\
R7 & COB & 169 & $-11,24$ & 0,151 & 15,41 & 0,97 \\
R8 & PL/1 & 26 & 7,87 & 0,107 & 12,83 & 0,87 \\
\hline
\end{tabular}

Table 2 Parabolic regression of TD against $\mathrm{N}_{1}$

Regression curve

\begin{tabular}{llcccccr} 
Appl. & Lang. & N. pgm. & Const. term & Linear term & Square term & Std. err. Corr. degr. \\
\hline R7 & COB & 169 & $-2,7$ & 0,123 & $1,5810^{-5}$ & 14,98 & 0,97
\end{tabular}

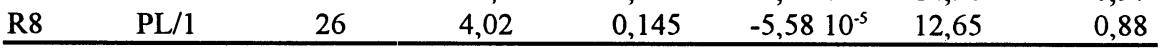

We are planning to use these models during the late phases of the life cycle of an application.

During the development of a new module for an existing application, we can use the model to predict the maximum number of decisions it can contain, and therefore we can keep under control the increase of computational complexity, which means to control the number of nodes in the control graph and hence the minimal number of independent paths that should be tested (small bugs in bank software can cost big money).

We can also avoid the 'unnecessary complexity' built into a solution but not required by the basic properties of the problem : if the value of TD predicted by the model is significantly lower than the measured TD, it is possible that the program could be rewritten in a simpler way (for instance, applying distributive properties to logical operators).

The programs in an application which are not so well represented by its model are those showing an apparently odd relationship between $\mathrm{N}_{1}$ and TD. These programs, especially if their values are higher than those given by the model, could be difficult to understand and to maintain, and more error-prone than the others. 
We measure the 'oddity degree' of a program within an application in standard deviation unit, i.e. using the ratio

$$
\mathrm{OD}=\left(\mathrm{TD}-\mathrm{TD}_{\text {mode }}\right) / \text { standard deviation }
$$

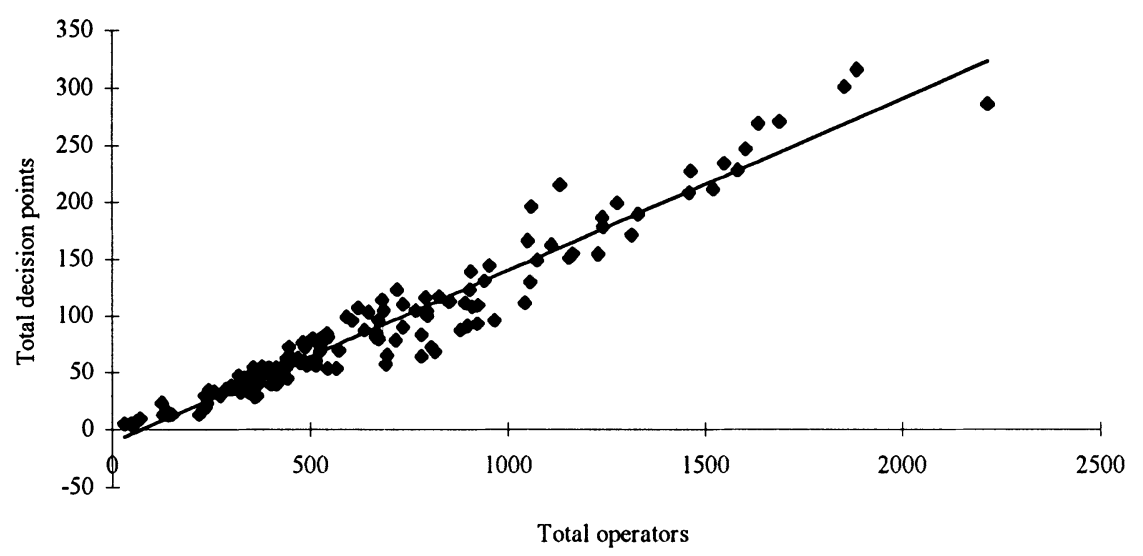

Figure 2 Total decision points against total operators for application R7 (Cobol). The chart shows the data distribution and the regression line.

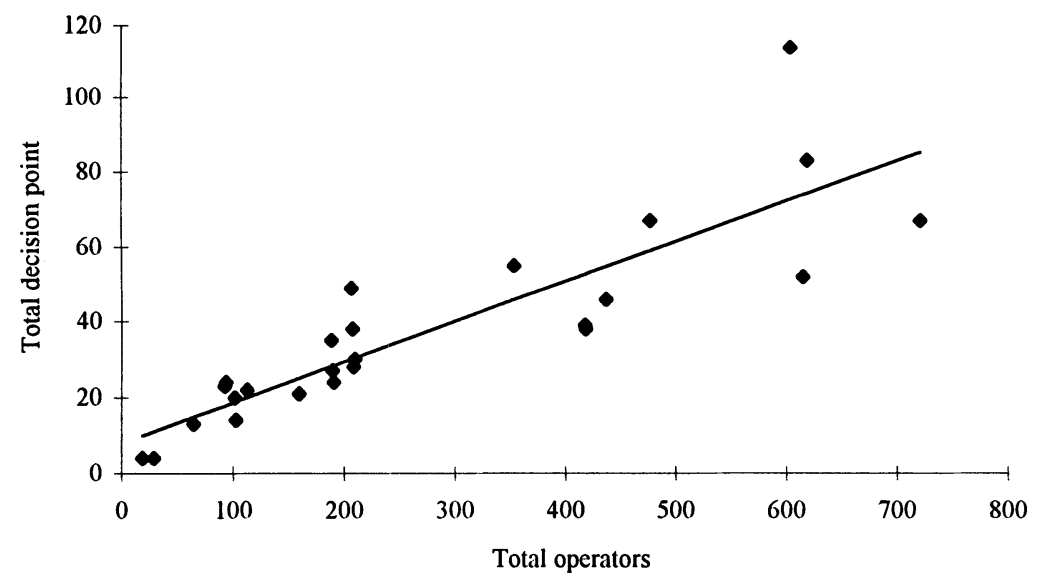

Figure 3 Total decision points against total operators for application R8 (PL/1). The chart shows the data distribution and the regression line. 
The three threshold values for OD were established in a way slightly different (though almost equivalent) from the other indexes.

If the program is less complex than what predicted by the model, OD will be negative, and the program will be considered acceptable, just as if it shows positive values lower than $1:$ the line parallel to the model and set at a distance equal to standard deviation is called 'acceptability line'.

Programs with values greater or equal to 1 but lower than 2 are considered as 'needing improvement'; the line as distant from the model as twice the standard deviation is called the 'improvement line'.

Programs with values greater or equal 2 but lower than 3 are considered 'critical'; the corresponding line is the 'critical line'.

Programs rated more than 3 are considered 'dangerous'; they can be placed anywhere beyond the critical line.

We defined another couple of ratios to measure the computational complexity even when the number of programs in the application is too low to reliably evaluate OD.

The first ratio is the average number of logical operators for decisional statements : it can provide an indication of the (average) complexity of the single decisional statement. A high ratio should lead the programmer to revise his code.

The second ratio is $C R=T D / N_{1}$. Since it is normalised with respect to one of the possible measures of size of the programs, it is convenient for comparison among different programs.

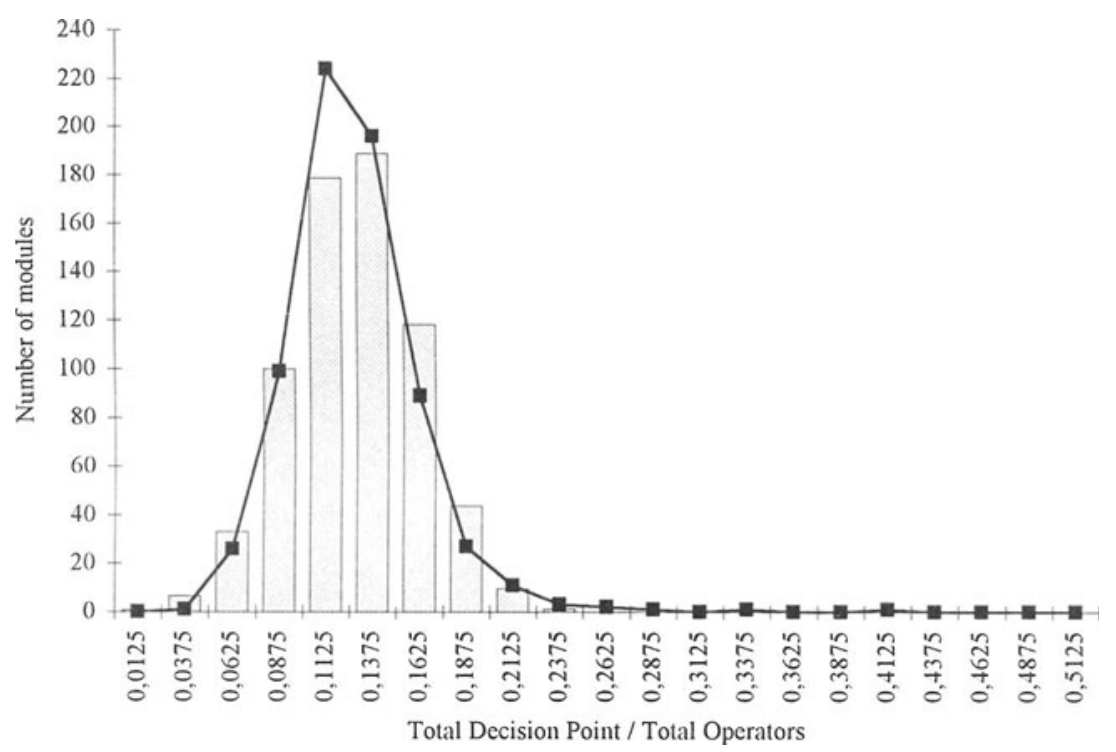

Figure 4 Frequency distribution of CR (line). The bars represent the normal distribution.

The frequency distribution of CR is rather close to normal (see Figure 4) and it shows a high concentration in the interval $[0.1,0.2[$, the same of the linear term of application models. 
During our study, we did not find values higher than 0.45 ; the $60 \%$ of the programs has a value behind 0.125 and the $50 \%$ a value behind 0.15 . Thus, even if the model is not available, or if it shows a low coefficient of correlation, a program with CR greater than 0.15 should anyway be regarded as 'suspect'.

\section{MANAGING QUALITY DATA : A SOFTWARE APPLICATION}

We already mentioned the software written to collect and analyse data obtained from source code. Its key capabilities are :

- the evaluation of the complexity model of the applications;

- the capability to store up to 99 values of each index, thus maintaining the history of programs and applications quality;

- the availability of the statistical analysis tool for setting thresholds and evaluating the frequency distributions of indicators.

The real purpose of the application is to give users something going 'beyond numbers' : all software analysers attach numbers to software, but usually they do not help in interpreting them.

This software has been by now released to technical users (project leaders and programmers) and we are beginning to collect data on a larger scale.

This phase has already lead to some adjustments, especially to threshold values; besides, we have got a number of suggestions about new possible metrics: our users seem very interested in nested control structures sensitivity, data complexity and in spotting the use of 'prohibited keywords' such as the COBOL statements GOTO or ALTER.

\section{CONCLUSIONS}

We could see that :

- on line programs tend to show higher complexity and size than batch ones, essentially because of input data validation;

- most of recent written programs have a size much smaller than older ones, due to the use of functional decomposition techniques;

- objects reusing and availability of 'skeleton programs' reduces the number of distinct operators $n_{1}$;

- high values of $n_{1}$ often indicate complex mathematical evaluations or string manipulations;

- anomalously high values of OD (i.e. 15) often indicate bad coding techniques, for instance long chains of explicit tests ('if $\mathrm{A}=24$ or $\mathrm{A}=25$ or $\mathrm{A}=\ldots$..) instead of more compact and performing table searches; OD can be also high (about 6 or 8 ) for huge programs belonging to an application whose other programs are much smaller; 
- very high values of program length (beyond 20000 words) often indicate that the program is made up by 'copy' or 'include' statements even in the non-declarative section, or that it has been split in parts because it exceeded the maximum size allowed by the editor; this makes the source program difficult to read and to control;

- code generators and reusable software show (of course!) a very high complexity and a strong ratio of logical operators, but this software is well represented by the model (no programs with OD higher than 3.5 );

- size, complexity and percentage of logical operators show rather clearly programs age.

It has to be noticed that, due to their size, extremely small programs (less than 20 operators) often result in extremely high values of CR, though they are very easy to read and to maintain.

Some of our users were a little disappointed : they observed that we rated as 'big' or 'complex' those programs which they already 'knew' to be 'big' and 'complex'. To begin with, this means that our models are reliable, and that estimation of effort and costs made using our data will probably be correct. Besides, our users did not ' $k n o w$ ' that the program were complex. They ' $f e l t$ ' it was so. Measuring software makes possible to replace 'traditional gutfeeling guesstimate' (DeMarco, 1982) with consistent forecasting obtained using common methods and evaluation tools and based on historical data.

\section{ACKNOWLEDGEMENTS}

The author gratefully thanks Dr. Ezio Ajmar (Società Italiana Avionica, Turin) for his many helpful suggestions and the extremely accurate review.

\section{REFERENCES}

Belady, L. A. (1983) Complexity of large systems, in Software Metrics : An analysis and Evaluation (ed. A. Perlis, F. Sayward, and M. Shaw), The MIT Press, Cambridge, Massachusets.

Chen, E. T. (1978) Program complexity and programmer productivity. IEEE Trans. Software Eng., SE-4 3,187-194.

Davis J.S. and LeBlanc R.J. (1988) A Study of the Applicability of Complexity Measures. IEEE Trans. Software Eng., 14 9, 1366-1372.

DeMarco, T. (1982) Controlling Software Projects : Management, Measurement and Estimation. N.J. Prentice Hall, Englewood Cliffs.

Grady, R.B. and Caswell, D.L. (1987) Software Metrics : Estabilishing a Company-Wide program. Prentice Hall.

Halstead, M.H. (1977) Elements of Software Science. Elsevier Nort-Holland, New York.

McCabe, T.J. (1976) A complexity measure. IEEE Trans. Software Eng., SE-2 4, 308-320.

Magg. Gen. tec. Mondino, Ten. Col. tec. Mele, Magg. tec. Messina and Cap. tec. Cascella (1994) L'esercito verso il controllo dell'ambiente di produzione del software militare, in Atti Ufficiali del CQS"94, Etnoteam - Nomos Ricerca.

Munson J.C. and Khoshgoftaar T.M. (1992) The detection of Fault-Prone Programs. IEEE 
Trans. Software Eng., 185 423-432.

Perlis, A. and Sayward, F. and Shaw, M. (1983) Software Metrics : An analysis and Evaluation. The MIT Press, Cambridge, Massachusets.

Sellers, B. H. (1992) Modularization and McCabe 's cyclomatic complexity. Comm. ACM, 35 12, 17-19.

Stroud, J.M. (1967) The fine structure of psychological time. New York Academy of Science Annals, 138(2) 623 - 631 .

Weyuker, E.J. (1988) Evaluating Software Complexity Measures. IEEE Trans. Software Eng., 14 9, 1357-1365.

\section{BIOGRAPHY}

Daniela Cellino received the degree in Mathematics from Università di Torino in 1982. Since then, she has been working on software development projects for insurance companies and banks. Actually, she has been the team leader of the group which designed and developed the teller support software used in front and back office activities in all branches of one of the most important Italian banks (about 10,000 workstations in the whole country). She is currently responsible for Software Engineering activities in the Turin branch of Banksiel S.p.A. (Finsiel Group), where she dealt for the past three years with software measurement activities. 